\title{
Variable-length Codes for Data Compression
}


David Salomon

Variable-length Codes

for Data Compression

黛 Springer 


\author{
Professor David Salomon (emeritus) \\ Computer Science Department \\ California State University \\ Northridge, CA 91330-8281 \\ USA \\ email: david.salomon@csun.edu
}

British Library Cataloguing in Publication Data

A catalogue record for this book is available from the British Library

Library of Congress Control Number:

ISBN 978-1-84628-958-3

e-ISBN 978-1-84628-959-0

Printed on acid-free paper.

(c) Springer-Verlag London Limited 2007

Apart from any fair dealing for the purposes of research or private study, or criticism or review, as permitted under the Copyright, Designs and Patents Act 1988, this publication may only be reproduced, stored or transmitted, in any form or by any means, with the prior permission in writing of the publishers, or in the case of reprographic reproduction in accordance with the terms of licences issued by the Copyright Licensing Agency. Enquiries concerning reproduction outside those terms should be sent to the publishers.

The use of registered names, trademarks, etc. in this publication does not imply, even in the absence of a specific statement, that such names are exempt from the relevant laws and regulations and therefore free for general use.

The publisher makes no representation, express or implied, with regard to the accuracy of the information contained in this book and cannot accept any legal responsibility or liability for any errors or omissions that may be made.

987765543221

Springer Science+Business Media

springer.com 
To the originators and developers of the codes. Apostolico, Capocelli, Elias, Fenwick, Fraenkel, Golomb, Huffman, Klein, Pigeon, Rice, Stout, Tsai, Tunstall, Villasenor, Wang, Wen, Wu, Yamamoto, and others.

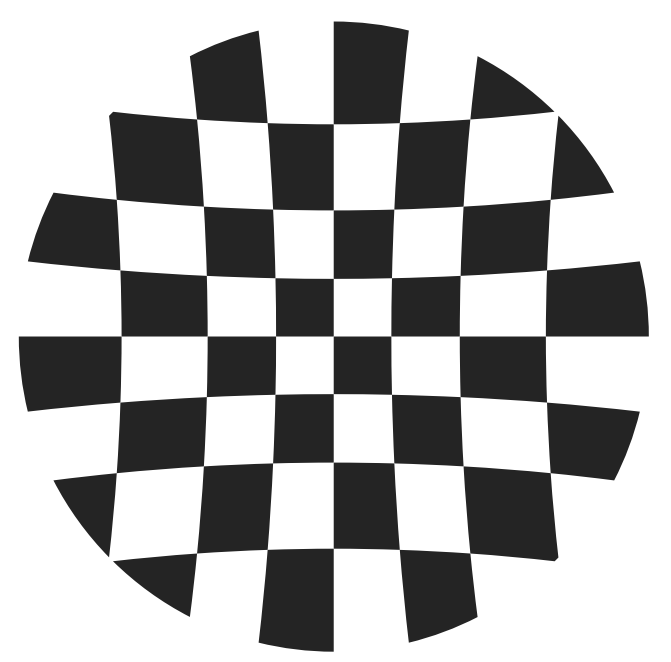

To produce a mighty book, you must choose a mighty theme. -Herman Melville 


\section{Preface}

The dates of most of the important historical events are known, but not always very precisely. We know that Kublai Khan, grandson of Ghengis Khan, founded the Yuan dynasty in 1280 (it lasted until 1368), but we don't know precisely (i.e., the month, day and hour) when this act took place. A notable exception to this state of affairs is the modern age of telecommunications, a historical era whose birth is known precisely, up to the minute. On Friday, 24 May 1844, at precisely 9:45 in the morning, Samuel Morse inaugurated the age of modern telecommunications by sending the first telegraphic message in his new code. The message was sent over an experimental line funded by the American Congress from the Supreme Court chamber in Washington, DC to the B \& O railroad depot in Baltimore, Maryland. Taken from the Bible (Numbers 23:23), the message was "What hath God wrought?" It had been suggested to Morse by Annie Ellsworth, the young daughter of a friend. It was prerecorded on a paper tape, was sent to a colleague in Baltimore, and was then decoded and sent back by him to Washington. An image of the paper tape can be viewed at [morse-tape 06].

Morse was born near Boston and was educated at Yale. We would expect the inventor of the telegraph (and of such a sophisticated code) to have been a child prodigy who tinkered with electricity and gadgets from an early age (the electric battery was invented when Morse was nine years old). Instead, Morse became a successful portrait painter with more than 300 paintings to his credit. It wasn't until 1837 that the 46-year-old Morse suddenly quit his painting career and started thinking about communications and tinkering with electric equipment. It is not clear why he made such a drastic career change at such an age, but it is known that two large, wall-size paintings that he made for the Capitol building in Washington, DC were ignored by museum visitors and rejected by congressmen. It may have been this disappointment that gave us the telegraph and the Morse code.

Given this background, it is easy to imagine how the 53-year-old Samuel Morse felt on that fateful day, Friday, 24 May 1844, as he sat hunched over his mysterious 
apparatus, surrounded by a curious crowd of onlookers, some of whom had only a vague idea of what he was trying to demonstrate. He must have been very anxious, because his telegraph project, his career, and his entire future depended on the success of this one test. The year before, the American Congress awarded him $\$ 30,000$ to prepare this historical test and prove the value of the electric telegraph (and thus also confirm the ingenuity of yankees), and here he is now, dependent on the vagaries of his batteries, on the new, untested 41-mile-long telegraph line, and on a colleague in Baltimore.

Fortunately, all went well. The friend in Baltimore received the message, decoded it, and resent it within a few minutes, to the great relief of Morse and to the amazement of the many congressmen assembled around him.

The Morse code, with its quick dots and dashes (Table 1), was extensively used for many years, first for telegraphy, and beginning in the $1890 \mathrm{~s}$, for early radio communications. The development of more advanced communications technologies in the 20th century displaced the Morse code, which is now largely obsolete. Today, it is used for emergencies, for navigational radio beacons, land mobile transmitter identification, and by continuous wave amateur radio operators.

\begin{tabular}{|c|c|c|c|c|c|c|c|}
\hline A &.- & $\mathrm{N}$ & - . & 1 &.---- & Period &...--- \\
\hline B & $-\ldots$ & $\mathrm{O}$ & --- & 2 & $\ldots---$ & Comma &..---- \\
\hline $\mathrm{C}$ &.-- . & $\mathrm{P}$ &.-- & 3 & $\ldots--$ & Colon & $--\ldots$ \\
\hline $\mathrm{Ch}$ & ---- & Q &.-- & 4 & $\ldots{ }_{-}^{-}$ & Question mark & $\ldots--\ldots$ \\
\hline $\mathrm{D}$ & $-\ldots$ & $\mathrm{R}$ &.- & 5 & $\ldots \ldots$ & Apostrophe &.---- \\
\hline $\mathrm{E}$ & . & $\mathrm{S}$ & $\ldots$ & 6 & $-\ldots$ & Hyphen & $-\ldots-$ \\
\hline $\mathrm{F}$ & $\ldots-$ & $\mathrm{T}$ & - & 7 & $--\ldots$ & Dash & $-\ldots-$ \\
\hline G & -- & $\mathrm{U}$ & $\ldots-$ & 8 & $---\ldots$ & Parentheses &..---- \\
\hline $\mathrm{H}$ & $\ldots$ & $\mathrm{V}$ & $\ldots-$ & 9 & ---- & Quotation marks &..-- \\
\hline I & . & W &.-- & 0 & ----- & & \\
\hline $\mathrm{J}$ &.--- & $\mathrm{X}$ & $-\ldots-$ & & & & \\
\hline K &.-- & $\mathrm{Y}$ &.--- & & & & \\
\hline $\mathrm{L}$ &.$-\ldots$ & $\mathrm{Z}$ & $--\ldots$ & & & & \\
\hline M & -- & & & & & & \\
\hline
\end{tabular}

Table 1: The Morse Code for English.

Our interest in the Morse code is primarily with a little-known aspect of this code. In addition to its advantages for telecommunications, the Morse code is also an early example of text compression. The various dot-dash codes developed by Morse (and possibly also by his associate, Alfred Vail) have different lengths, and Morse intuitively assigned the short codes (a single dot and a single dash) to the letters $\mathrm{E}$ and $\mathrm{T}$, the longer, four dots-dashes, he assigned to Q, X, Y, and Z. The even longer, five dotsdashes codes, were assigned to the 10 digits, and the longest codes (six dots and dashes) became those of the punctuation marks. Morse also specified that the signal for error is eight consecutive dots, in response to which the receiving operator should delete the last word received. 
It is interesting to note that Morse was not the first to think of compression (in terms of time saving) by means of a code. The well-known Braille code for the blind was developed by Louis Braille in the 1820s and is still in common use today. It consists of groups (or cells) of $3 \times 2$ dots each, embossed on thick paper. Each of the six dots in a group may be flat or raised, implying that the information content of a group is equivalent to six bits, resulting in 64 possible groups. The letters, digits, and common punctuation marks do not require all 64 codes, which is why the remaining groups may be used to code common words - such as and, for, and of - and common strings of letters - such as ound, ation, and th.

The Morse code has another feature that makes it relevant to us. Because the individual codes have different lengths, there must be a way to identify the end of a code. Morse solved this problem by requiring accurate relative timing. If the duration of a dot is taken to be one unit, then that of a dash is three units, the space between the dots and dashes of one character is one unit, the space between characters is three units, and the interword space is six units (five for automatic transmission). This book is concerned with the use of variable-length codes to compress digital data. With these codes, it is important not to have any extra spaces. In fact, there is no such thing as a space, because computers use only zeros and 1's. Thus, when a string of data symbols is compressed by assigning short codes (that are termed "codewords") to the symbols, the codewords (whose lengths vary) are concatenated into a long binary string without any spaces or separators. Such variable-length codes must therefore be designed to allow for unambiguous reading. Somehow, the decoder should be able to read bits and identify the end of each codeword. Such codes are referred to as uniquely decodable or uniquely decipherable (UD).

Variable-length codes have become important in many areas of computer science. This book is a survey of this important topic. It presents the principles underlying this type of codes and describes the important classes of variable-length codes. Many examples illustrate the applications of these codes to data compression. The book is devoted to the codes, which is why it describes very few actual compression algorithms. Notice that many important (and some not so important) methods, algorithms, and techniques for compressing data are described in detail in [Salomon 06].

The term representation is central to our discussion. A number can be represented in decimal, binary, or any other number base (or number system, see Section 2.18). Mathematically, a representation is a bijection (or a bijective function) of an infinite, countable set $S_{1}$ of strings onto another set $S_{2}$ of strings (in practice, $S_{2}$ consists of binary strings, but it may also be ternary or based on other number systems), such that any concatenation of any elements of $S_{2}$ is UD. The elements of $S_{1}$ are called data symbols and those of $S_{2}$ are codewords. Set $S_{1}$ is an alphabet and set $S_{2}$ is a code. An interesting example is the standard binary notation. We normally refer to it as the binary representation of the integers, but according to the definition above it is not a representation because it is not UD. It is easy to see, for example, that a string of binary codewords that starts with 11 can be either two consecutive 1's or the code of 3 .

A function $f: X \Rightarrow Y$ is said to be bijective, if for every $y \in Y$, there is exactly one $x \in X$ such that $f(x)=y$. 
Figure 3.19 and Table 3.22 list several variable-length UD codes assigned to the 26 letters of the English alphabet.

This book is aimed at readers who have a basic knowledge of data compression and who want to know more about the specific codes used by the various compression algorithms. The necessary mathematical background includes logarithms, polynomials, a bit of calculus and linear algebra, and the concept of probability. This book is not intended as a guide to software implementors and has no programs. Errors, mistypes, comments, and questions should be sent to the author's email address below.

It is my pleasant duty to acknowledge the substantial help and encouragement I have received from Giovanni Motta and Cosmin Truţa and for their painstaking efforts. They read drafts of the text, found many errors and misprints, and provided valuable comments and suggestions that improved this book and made it what it is. Giovanni also wrote part of Section 2.12.

If, by any chance, I have omitted anything more or less proper or necessary, I beg forgiveness, since there is no one who is without fault and circumspect in all matters.

-Leonardo Fibonacci, Libe Abaci (1202)

dsalomon@csun.edu

David Salomon

The Preface is the most important part of the book. Even reviewers read a preface.

-Philip Guedalla

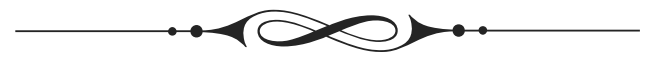




\section{Contents}

Preface

vii

Introduction

1 Basic Codes

1.1 Codes, Fixed- and Variable-Length

1.2 Prefix Codes

12

1.3 VLCs, Entropy, and Redundancy

13

1.4 Universal Codes

1.5 The Kraft-McMillan Inequality

1.6 Tunstall Code

21

1.7 Schalkwijk's Coding

1.8 Tjalkens-Willems V-to-B Coding 28

1.9 Phased-In Codes 31

1.10 Redundancy Feedback (RF) Coding 33

1.11 Recursive Phased-In Codes 37

1.12 Self-Delimiting Codes 40

1.13 Huffman Coding 42

2 Advanced Codes

$2.1 \quad$ VLCs for Integers

69

$2.2 \quad$ Start-Step-Stop Codes

$2.3 \quad$ Start/Stop Codes

$2.4 \quad$ Elias Codes

2.5 Levenstein Code 80

2.6 Even-Rodeh Code 81

2.7 Punctured Elias Codes 82

$2.8 \quad$ Other Prefix Codes 83

2.9 Ternary Comma Code 86

2.10 Location Based Encoding (LBE) 87

2.11 Stout Codes $\quad 89$

2.12 Boldi-Vigna $(\zeta)$ Codes $\quad 91$ 
2.13 Yamamoto's Recursive Code 94

2.14 VLCs and Search Trees 97

2.15 Taboo Codes 100

2.16 Wang's Flag Code 105

2.17 Yamamoto Flag Code 106

2.18 Number Bases 110

2.19 Fibonacci Code 112

2.20 Generalized Fibonacci Codes 116

2.21 Goldbach Codes 120

2.22 Additive Codes 126

2.23 Golomb Code 129

2.24 Rice Codes 136

2.25 Subexponential Code 138

2.26 Codes Ending with "1" 139

3 Robust Codes 143

3.1 Codes For Error Control 143

3.2 The Free Distance 149

3.3 Synchronous Prefix Codes 150

3.4 Resynchronizing Huffman Codes 156

3.5 Bidirectional Codes 159

3.6 Symmetric Codes 168

3.7 VLEC Codes 170

$\begin{array}{ll}\text { Summary and Unification — } & 177\end{array}$

Bibliography — 179

$\begin{array}{lr}\text { Index } & 187\end{array}$

An adequate table of contents serves as a synopsis or headline display of the design or structural pattern of the body of the report.

-D. E. Scates and C. V. Good, Methods of Research

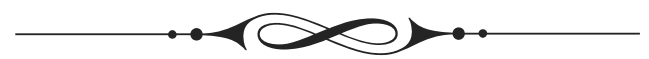




\section{Introduction}

The discipline of data compression has its origins in the 1950s and 1960s and has experienced rapid growth in the 1980s and 1990s. Currently, data compression is a vast field encompassing many approaches and techniques. A student of this field realizes quickly that the various compression algorithms in use today are based on and require knowledge of diverse physical and mathematical concepts and topics, some of which are included in the following, incomplete list: Fourier transforms, finite automata, Markov processes, the human visual and auditory systems - statistical terms, distributions, and concepts-Unicode, XML, convolution, space-filling curves, Voronoi diagrams, interpolating polynomials, Fibonacci numbers, polygonal surfaces, data structures, the Vandermonde determinant, error-correcting codes, fractals, the Pascal triangle, fingerprint identification, and analog and digital video.

Faced with this complexity, I decided to try and classify in this short introduction most (but not all) of the approaches to data compression in four classes as follows: (1) block-to-block codes, (2) block-to-variable codes, (3) variable-to-block codes, and (4) variable-to-variable codes (the term "fixed" is sometimes used instead of "block"). Other approaches to compression, such as mathematical transforms (orthogonal or wavelet) and the technique of arithmetic coding, are not covered here. Following is a short description of each class.

- Block-to-block codes constitute a class of techniques that input $n$ bits of raw data at a time, perform a computation, and output the same number of bits. Such a process results in no compression; it only transforms the data from its original format to a format where it becomes easy to compress. Thus, this class consists of transforms. The discrete wavelet, discrete cosine, and linear prediction are examples of transforms that are commonly used as the first step in the compression of various types of data. Here is a short description of linear prediction.

Audio data is common in today's computers. We all have mp3, FLAC, and other types of compressed audio files in our computers. A typical lossless audio compression technique consists of three steps. (1) The original sound is sampled (digitized). (2) The audio samples are converted, in a process that employs linear prediction, to small numbers called residues. (3) The residues are replaced by variable-length codes. The 
last step is the only one that produces compression.

Linear prediction of audio samples is based on the fact that most audio samples are similar to their near neighbors. One second of audio is normally converted to many thousands of audio samples (44,100 samples per second is typical), and adjacent samples tend to be similar because sound rarely varies much in pitch or frequency during one second. If we denote the current audio sample by $s(t)$, then linear prediction computes a predicted value $\hat{s}(t)$ from the $p$ immediately-preceding samples by a linear combination of the form

$$
\hat{s}(t)=\sum_{i=1}^{p} a_{i} s(t-i) .
$$

Parameter $p$ depends on the specific algorithm and many also be user controlled. Parameters $a_{i}$ are linear coefficients that are also determined by the algorothm.

If the prediction is done properly, the difference (which is termed residue or residual) $e(t)=s(t)-\hat{s}(t)$ will almost always be a small (positive or negative) number, although in principle it could be about as large as $s(t)$ or $-s(t)$. The difference between the various linear prediction methods is in the number $p$ of previous samples that they employ and in the way they determine the linear coefficients $a_{i}$.

- Block-to-variable codes are the most important of the four types discussed here. Each symbol of the input alphabet is assigned a variable-length code according to its frequency of occurrence (or, equivalently, its probability) in the data. Compression is achieved if short codes are assigned to commonly-occurring (high probability) symbols and long codes are assigned to rare symbols. Many statistical compression methods employ this type of coding, most notably the Huffman method (Section 1.13). The difference between the various methods is mostly in how they compute or estimate the probabilities of individual data symbols. There are three approaches to this problem, namely static codes, a two-pass algorithm, and adaptive methods.

Static codes. It is possible to construct a set of variable-length codes and permanently assign each code to a data symbol. The result is a static code table that is built into both encoder and decoder. To construct such a table, the developer has to analyze large quantities of data and determine the probability of each symbol. For example, someone who develops a compression method that employs this approach to compress text, has to start by selecting a number of representative "training" documents, count the number of times each text character appears in those documents, compute frequencies of occurrence, and use this fixed, static statistical model to assign variable-length codewords to the individual characters. A compression method based on a static code table is simple, but the results (the compression ratio for a given text file) depend on how much the data resembles the statistics of the training documents.

A two-pass algorithm. The idea is to read the input data twice. The first pass simply counts symbol frequencies and the second pass performs the actual compression by replacing each data symbol with a variable-length codeword. In between the two passes, the code table is constructed by utilizing the symbols' frequencies in the particular data being compressed (the statistical model is taken from the data itself). Such a method features very good compression, but is slow because reading a file from an input device, even a fast disk, is slower than memory-based operations. Also, the code table is constructed individually for each data file being compressed, so it has to be included 
in the compressed file, for the decoder's use. This reduces the compression ratio but not significantly, because a code table typically contains one variable-length code for each of the 128 ASCII characters or for each of the 256 8-bit bytes, so its total length is only a few hundred bytes.

An adaptive method starts with an empty code table, or with a tentative table, and modifies the table as more data is read and processed. Initially, the codes assigned to the data symbols are inappropriate and are not based on the (unknown) probabilities of the data symbols. But as more data is read, the encoder acquires better statistics of the data and exploits it to improve the codes (the statistical model adapts itself gradually to the data that is being read and compressed). Such a method has to be designed to permit the decoder to mimic the operations of the encoder and modify the code table in lockstep with it.

A simple statistical model assigns variable-length codes to symbols based on symbols' probabilities. It is possible to improve the compression ratio significantly by basing the statistical model on probabilities of pairs or triplets of symbols (digrams and trigrams), instead of probabilities of individual symbols. The result is an $n$-order statistical compression method where the previous $n$ symbols are used to predict (i.e., to assign a probability to) the current symbol. The PPM (prediction by partial matching) and DMC (dynamic Markov coding) methods are examples of this type of algorithm.

It should be noted that arithmetic coding, an important statistical compression method, is included in this class, but operates differently. Instead of assigning codes to individual symbols (bits, ASCII codes, Unicodes, bytes, etc.), it assigns one long code to the entire input file.

- Variable-to-block codes is a term that refers to a large group of compression techniques where the input data is divided into chunks of various lengths and each chunk of data symbols is encoded by a fixed-size code. The most important members of this group are run-length encoding and the various LZ (dictionary-based) compression algorithms.

A dictionary-based algorithm saves bits and pieces of the input data in a special buffer called a dictionary. When the next item is read from the input file, the algorithm tries to locate it in the dictionary. If the item is found in the dictionary, the algorithm outputs a token with a pointer to the item plus other information such as the length of the item. If the item is not in the dictionary, the algorithm adds it to the dictionary (based on the assumption that once an item has appeared in the input, it is likely that it will appear again) and outputs the item either in raw format or as a special, literal token. Compression is achieved if a large item is replaced by a short token. Quite a few dictionary-based algorithms are currently known. They have been developed by many scientists and researchers, but are all based on the basic ideas and pioneering work of Jacob Ziv and Abraham Lempel, described in [Ziv and Lempel 77] and [Ziv and Lempel 78].

A well-designed dictionary-based algorithm can achieve high compression because a given item tends to appear many times in a data file. In a text file, for example, the same words and phrases may appear many times. Words that are common in the language and phrases that have to do with the topic of the text, tend to appear again and again. If they are kept in the dictionary, then more and more phrases can be replaced by tokens, thereby resulting in good compression. 
The differences between the various LZ dictionary methods are in how the dictionary is organized and searched, in the format of the tokens, in the way the algorithm handles items not found in the dictionary, and in the various improvements it makes to the basic method. The many variants of the basic LZ approach employ improving techniques such as a circular buffer, a binary search tree, variable-length codes or dynamic Huffman coding to encode the individual fields of the token, and other tricks of the programming trade. Sophisticated dictionary organization eliminates duplicates (each data symbol is stored only once in the dictionary, even if it is part of several items), implements fast search (binary search or a hash table instead of slow linear search), and may discard unused items from time to time in order to regain space.

The other important group of variable-to-block codes is run-length encoding (RLE). We know that data can be compressed because the common data representations are redundant, and one type of redundancy is runs of identical symbols. Text normally does not feature long runs of identical characters (the only examples that immediately come to mind are runs of spaces and of periods), but images, especially monochromatic (black and white) images, may have long runs of identical pixels. Also, an audio file may have silences, and even one-tenth of second worth of silence typically translates to 4,410 identical audio samples.

A typical run-length encoder identifies runs of the same symbol and replaces each run with a token that includes the symbol and the length of the run. If the run is shorter than a token, the raw symbols are output, but the encoder has to make sure that the decoder can distinguish between tokens and raw symbols.

Since runs of identical symbols are not common in many types of data, run-length encoding is often only one part of a larger, more sophisticated compression algorithm.

- Variable-to-variable codes is the general name used for compression methods that select variable-length chunks of input symbols and compress each chunk by replacing it with a variable-length code.

A simple example of variable-to-variable codes is run-length encoding combined with Golomb codes, especially when the data to be compressed is binary. Imagine a long string of 0's and 1's where one value (say, 0) occurs more often than the other value. This value is referred to as the more probable symbol (MPS), while the other value becomes the LPS. Such a string tends to have runs of the MPS and Section 2.23 shows that the Golomb codes are the best candidate to compress such runs. Each run has a different length, and the various Golomb codewords also have different lengths, turning this application into an excellent example of variable-to-variable codes.

Other examples of variable-to-variable codes are hybrid methods that consist of several parts. A hybrid compression program may start by reading a chunk of input and looking it up in a dictionary. If a match is found, the chunk may be replaced by a token, which is then further compressed (in another part of the program) by RLE or variablelength codes (perhaps Huffman or Golomb). The performance of such a program may not be spectacular, but it may produce good results for many different types of data. Thus, hybrids tend to be general-purpose algorithms that can deal successfully with text, images, video, and audio data.

This book starts with several introductory sections (Sections 1.1 through 1.6) that discuss information theory concepts such as entropy and redundancy, and concepts that 
are used throughout the text, such as prefix codes, complete codes, and universal codes.

The remainder of the text deals mostly with block-to-variable codes, although its first part deals with the Tunstall codes and other variable-to-block codes. It concentrates on the codes themselves, not on the compression algorithms. Thus, the individual sections describe various variable-length codes and classify them according to their structure and organization. The main techniques employed to design variable-length codes are the following:

- The phased-in codes (Section 1.9) are a slight extension of fixed-size codes and may contribute a little to the compression of a set of consecutive integers by changing the representation of the integers from fixed $n$ bits to either $n$ or $n-1$ bits (recursive phased-in codes are also described).

- Self-delimiting codes. These are intuitive variable-length codes - mostly due to Gregory Chaitin, the originator of algorithmic information theory - where a code signals its end by means of extra flag bits. The self-delimiting codes of Section 1.12 are inefficient and are not used in practice.

- Prefix codes. Such codes can be read unambiguously (they are uniquely decodable, or UD codes) from a long string of codewords because they have a special property (the prefix property) which is stated as follows: Once a bit pattern is assigned as the code of a symbol, no other codes can start with that pattern. The most common example of prefix codes are the Huffman codes (Section 1.13). Other important examples are the unary, start-step-stop, and start/stop codes (Sections 2.2 and 2.3, respectively).

- Codes that include their own length. One way to construct a UD code for the integers is to start with the standard binary representation of an integer and prepend to it its length $L_{1}$. The length may also have variable length, so it has to be encoded in some way or have its length $L_{2}$ prepended. The length of an integer $n$ equals approximately $\log n$ (where the logarithm base is the same as the number base of $n$ ), which is why such methods are often called logarithmic ramp representations of the integers. The most common examples of this type of codes are the Elias codes (Section 2.4), but other types are also presented. They include the Levenstein code (Section 2.5), EveRodeh code (Section 2.6), punctured Elias codes (Section 2.7), the ternary comma code (Section 2.9), Stout codes (Section 2.11), Boldi-Vigna (zeta) codes (Section 2.12), and Yamamoto's recursive code (Section 2.13).

- Suffix codes (codes that end with a special flag). Such codes limit the propagation of an error and are therefore robust. An error in a codeword affects at most that codeword and the one or two codewords following it. Most other variable-length codes sacrifice data integrity to achieve short codes, and are fragile because a single error can propagate indefinitely through a sequence of concatenated codewords. The taboo codes of Section 2.15 are UD because they reserve a special string (the taboo) to indicate the end of the code. Wang's flag code (Section 2.16) is also included in this category.

Note. The term "suffix code" is ambiguous. It may refer to codes that end with a special bit pattern, but it also refers to codes where no codeword is the suffix of another codeword (the opposite of prefix codes). The latter meaning is used in Section 3.5, in connection with bidirectional codes. 
- Flag codes. A true flag code differs from the suffix codes in one interesting aspect. Such a code may include the flag inside the code, as well as at its right end. The only example of a flag code is Yamamoto's code, Section 2.17.

- Codes based on special number bases or special number sequences. We normally use decimal numbers, and computers use binary numbers, but any integer greater than 1 can serve as the basis of a number system and so can noninteger (real) numbers. It is also possible to construct a sequence of numbers (real or integer) that act as weights of a numbering system. The most important examples of this type of variable-length codes are the Fibonacci (Section 2.19), Goldbach (Section 2.21), and additive codes (Section 2.22).

- The Golomb codes of Section 2.23 are designed in a special way. An integer parameter $m$ is selected and is used to encode an arbitrary integer $n$ in two steps. In the first step, two integers $q$ and $r$ (for quotient and remainder) are computed from $n$ such that $n$ can be fully reconstructed from them. In the second step, $q$ is encoded in unary and is followed by the binary representation of $r$, whose length is implied by the parameter $m$. The Rice code of Section 2.24 is a special case of the Golomb codes where $m$ is an integer power of 2. The subexponential code (Section 2.25) is related to the Rice codes.

- Codes ending with " 1 " are the topic of Section 2.26. In such a code, all the codewords end with a 1, a feature that makes them the natural choice in special applications.

- Variable-length codes are designed for data compression, which is why implementors select the shortest possible codes. Sometimes, however, data reliability is a concern, and longer codes may help detect and isolate errors. Thus, Chapter 3 discusses robust codes. Section 3.3 presents synchronous prefix codes. These codes are useful in applications where it is important to limit the propagation of errors. Bidirectional (or reversible) codes (Sections 3.5 and 3.6) are also designed for increased reliability by allowing the decoder to read and identify codewords either from left to right or in reverse.

The following is a short discussion of terms that are commonly used in this book.

- Source. A source of data items can be a file stored on a disk, a file that is input from outside the computer, text input from a keyboard, or a program that generates data symbols to be compressed or processed in some way. In a memoryless source, the probability of occurrence of a data symbol does not depend on its context. The term i.i.d. (independent and identically distributed) refers to a set of sources that have the same probability distribution and are mutually independent.

- Alphabet. This is the set of symbols that an application has to deal with. An alphabet may consist of the 128 ASCII codes, the 256 8-bit bytes, the two bits, or any other set of symbols.

- Random variable. This is a function that maps the results of random experiments to numbers. For example, selecting many people and measuring their heights is a random variable. The number of occurrences of each height can be used to compute the probability of that height, so we can talk about the probability distribution of the random variable (the set of probabilities of the heights). A special important case is a discrete random variable. The set of all values that such a variable can assume is finite or countably infinite. 
- Compressed stream (or encoded stream). A compressor (or encoder) compresses data and generates a compressed stream. This is often a file that is written on a disk or is stored in memory. Sometimes, however, the compressed stream is a string of bits that are transmitted over a communications line.

- The acronyms MSB and LSB refer to most-significant-bit and least-significant-bit, respectively.

- The notation $1^{i} 0^{j}$ indicates a bit string of $i$ consecutive 1 's followed by $j$ zeros.

Understanding is, after all, what science is all about-and science is a great deal more than mere mindless computation.

- Roger Penrose, Shadows of the Mind (1996)

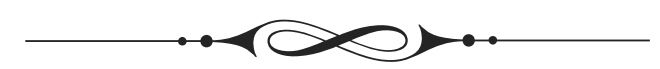

